\title{
Validation of Highly Accelerated Wave-CAIPI SWI Compared with Conventional SWI and T2*-Weighted Gradient Recalled-Echo for Routine Clinical Brain MRI at 3T
}

(D). Conklin, (D) M.G.F. Longo, (D) S.F. Cauley, (D). Setsompop, (D).G. González, (DP.W. Schaefer, (D).E. Kirsch, (D). Rapalino, and (iD).Y. Huang

\begin{abstract}
BACKGROUND AND PURPOSE: SWI is valuable for characterization of intracranial hemorrhage and mineralization but has long acquisition times. We compared a highly accelerated wave-controlled aliasing in parallel imaging (CAIPI) SWI sequence with 2 commonly used alternatives, standard SWI and T2*-weighted gradient recalled-echo (T2*W GRE), for routine clinical brain imaging at 3 T.

MATERIALS AND METHODS: A total of 246 consecutive adult patients were prospectively evaluated using a conventional SWI or T2*W GRE sequence and an optimized wave-CAIPI SWI sequence, which was 3-5 times faster than the standard sequence. Two blinded radiologists scored each sequence for the presence of hemorrhage, the number of microhemorrhages, and severity of motion artifacts. Wave-CAIPI SWI was then evaluated in head-to-head comparison with the conventional sequences for visualization of pathology, artifacts, and overall diagnostic quality. Forced-choice comparisons were used for all scores. Wave-CAIPI SWI was tested for superiority relative to T2*W GRE and for noninferiority relative to standard SWI using a $15 \%$ noninferiority margin.

RESULTS: Compared with T2*W GRE, wave-CAIPI SWI detected hemorrhages in more cases $(P<.001)$ and detected more microhemorrhages $(P<.001)$. Wave-CAIPI SWI was superior to T2*W GRE for visualization of pathology, artifacts, and overall diagnostic quality (all $P<.001)$. Compared with standard SWI, wave-CAIPI SWI showed no difference in the presence or number of hemorrhages identified. Wave-CAIPI SWI was noninferior to standard SWI for the visualization of pathology $(P<.001)$, artifacts $(P<.01)$, and overall diagnostic quality $(P<.01)$. Motion was less severe with wave-CAIPI SWI than with standard SWI $(P<.01)$.
\end{abstract}

CONCLUSIONS: Wave-CAIPI SWI provided superior visualization of pathology and overall diagnostic quality compared with T2*W GRE and was noninferior to standard SWI with reduced scan times and reduced motion artifacts.

ABBREVIATIONS: CAIPI = controlled aliasing in parallel imaging; GRE $=$ gradient recalled-echo; MARS $=$ Microbleed Anatomical Rating Scale

S WI is widely applied for clinical brain imaging due to its exquisite sensitivity for the detection and characterization of blood products, superior to that of conventional $\mathrm{T} 22^{\star}$-weighted gradient recalled-echo (T2*W GRE) imaging. ${ }^{1}$ SWI is useful in the evaluation of a broad range of pathology including vascular malformations, hemorrhagic or calcified neoplasms, vasculopathies, and neurodegenerative disorders associated with

Received March 5, 2019; accepted after revision September 9.

From the Department of Radiology (J.C., M.G.F.L., S.F.C., K.S., R.G.G., P.W.S., J.E.K., O.R., S.Y.H.), Massachusetts General Hospital, Boston, Massachusetts; Athinoula A. Martinos Center for Biomedical Imaging (S.F.C., K.S., S.Y.H.), Boston, Massachusetts; and Harvard-MIT Division of Health Sciences and Technology (K.S., S.Y.H.), Massachusetts Institute of Technology, Cambridge, Massachusetts.

J. Conklin and M.G.F. Longo contributed equally to this work.

This work was supported by the National Institutes of Health (P41 EB015896, R01 EB020613, UL 1TR002541), a Radiological Society of North America Research Resident Grant (S.Y.H.), and Siemens Healthineers. This work was conducted with support from Harvard Catalyst, Harvard Clinical and Translational Science Center (National Center for Advancing Translational Sciences, National Institutes of Health Award UL 1TR002541), and financial contributions from Harvard University and its affiliated academic health care centers. mineralization or brain iron accumulation. ${ }^{2,3}$ However, conventional SWI is associated with long acquisition times (typically $\geq 5$ minutes), ${ }^{1}$ which may contribute to motion artifacts ${ }^{4}$ and patient anxiety. ${ }^{5}$

Wave-controlled aliasing in parallel imaging (CAIPI) is a rapid acquisition approach that combines a corkscrew gradient trajectory with CAIPI shifts in the $k_{\mathrm{y}}$ and $k_{\mathrm{z}}$ directions to efficiently encode $k$-space and uniformly spread the voxel aliasing,

The content is solely the responsibility of the authors and does not necessarily represent the official views of Harvard Catalyst, Harvard University and its affiliated academic healthcare centers, or the National Institutes of Health.

Please address correspondence to J. Conklin, MD, MSc, Division of Neuroradiology, Department of Radiology, Massachusetts General Hospital, 55 Fruit St, GRB-273A, Boston, MA, 02114; e-mail: jconklin1@mgh.harvard.edu

\footnotetext{
- Indicates open access to non-subscribers at www.ajnr.org

三 Indicates article with supplemental on-line tables.

Indicates article with supplemental on-line photo.

http://dx.doi.org/10.3174/ajnr.A6295
} 
taking full advantage of the 3D coil sensitivity information to provide high acceleration factors with negligible artifacts and g-factor penalty. ${ }^{6,7}$ SWI is well-suited for this aggressive acceleration strategy due to the intrinsically high contrast between the pathology of interest (which manifests as a signal void) and background brain tissue. The resulting decrease in acquisition time may facilitate broader clinical application of SWI, especially in motionprone populations (eg, children, elderly, and acutely ill patients). Wave-CAIPI has shown the potential to accelerate susceptibilityweighted acquisitions in healthy volunteers ${ }^{8,9}$ but has not been systematically evaluated in a clinical setting.

The goal of this study was to compare a highly accelerated SWI sequence based on wave-CAIPI (wave-SWI) with 2 commonly used alternatives, conventional $3 \mathrm{D}-\mathrm{SWI}$ and $2 \mathrm{D} \mathrm{T} 2^{\star} \mathrm{W}$ GRE. We hypothesized that wave-SWI would be superior to $\mathrm{T} 2{ }^{*} \mathrm{~W}$ GRE for visualization of pathology and overall diagnostic quality in a similar scan time and noninferior to the standard SWI sequence with a 3 - to 5 -fold reduction in scan time.

\section{MATERIALS AND METHODS \\ Subjects and Study Design}

A prospective comparative study was performed at a single institution (Massachusetts General Hospital, Boston, MA). Consecutive adult patients (older than 19 years of age; $n=246$ ) undergoing clinical brain MR imaging were prospectively evaluated, including both inpatient and outpatient examinations. Imaging was performed on a 3T Magnetom Prisma scanner from March to June 2018 and on a 3T Magnetom Skyra MR imaging scanner from May to June 2018 (Siemens, Erlangen, Germany). There were no exclusion criteria beyond those for routine clinical MR imaging. The study was Health Insurance Portability and Accountability Act-compliant and approved by our institutional review board. Verbal consent was obtained before MR imaging. Written consent was waived by the institutional review board. Demographics of the study subjects and clinical indications for MR imaging are shown in On-line Table 1.

\section{Wave-CAIPI SWI Pulse Sequence and Reconstruction}

Wave-SWI was implemented using a work-in-progress dual-echo $3 \mathrm{D}$ gradient-echo pulse sequence ${ }^{6}$ (WIP1058C; Siemens). On-line reconstruction was performed using an autocalibrated procedure for simultaneous estimation of the parallel imaging reconstruction and true $k$-space trajectory (which accounts for potential gradient hardware imperfections), ${ }^{7}$ with a reconstruction time of approximately 60 seconds. This included phase unwrapping of the reconstructed multiecho data and a weighted combination that accounted for the TE phase evolution. The standard vendor SWI processing was then performed to produce high-pass-filtered phase images and associated SWI. Pulse sequence parameters could not be exactly matched between the wave-SWI and standard SWI sequences due to vendor constraints on the available parameter options, but they were approximated as closely as possible within the allowable range of parameter values (On-line Table 2).

\section{MR Imaging Protocol}

Each patient underwent brain MR imaging on 1 of two 3T MR imaging scanners using commercially available 20- and 32-channel receiver coil arrays (Siemens). Standard institutional brain MR imaging protocols were selected by the radiologist on the basis of the provided clinical indication. Each scan included a conventional magnetic susceptibility-weighted sequence (either standard SWI or T2*W GRE, decided at the discretion of the protocoling radiologist based on the clinical indication) and a highly accelerated wave-SWI sequence performed immediately before or after the conventional susceptibility-weighted sequence. Acceleration factors of $R=3 \times 2$ and $R=3 \times 3$ were chosen on the 20-channel and 32-channel coils, respectively, to balance scan time with an acceptable SNR for each coil configuration. ${ }^{10}$ The acquisition order alternated on a weekly basis to control for the possible effect of increased patient motion on the later sequence. A summary of the magnetic susceptibility-weighted sequence parameters is provided in On-line Table 2.

\section{Image Evaluation}

A semiquantitative grading system based on predetermined criteria was used to compare wave-SWI with the conventional susceptibility-weighted sequence acquired for each patient (standard SWI or T2*W GRE). The DICOM datasets were anonymized and transferred to an independent workstation. Blinded to patient information and protocol type, 2 neuroradiologists (O.R. and S.Y.H.) with 17 and 7 years of experience, respectively, independently reviewed all images in randomized order. To obtain optimal visualization, adjustments of window widths and levels were allowed. Only the magnetic susceptibility-weighted sequences were evaluated.

To provide a comprehensive assessment of the diagnostic performance of the wave-SWI and conventional susceptibilityweighted sequences, we divided the review sessions into an individual image series analysis ("individual analysis"), in which images obtained using each sequence were presented in isolation, and a head-to-head image series analysis ("head-to-head analysis"), in which images obtained using the wave-SWI and standard magnetic susceptibility-weighted sequence were presented sideby-side with randomly selected left and right screen positions. The individual analysis was conducted in 2 sessions so that only 1 sequence per patient was presented to the reviewer in each session. The individual analysis sessions were separated by at least 2 weeks to minimize memory bias.

In the individual analysis, reviewers evaluated the following: the presence of hemorrhage, the number and location of the cerebral microhemorrhages (if present), the degree of motion, and whether the images were of diagnostic quality (yes or no). Motion was scored on a predefined 4-point scale. To minimize subjectivity, representative images of each motion score were available to reviewers during the evaluation (On-line Figure). Microhemorrhages were defined according to the Microbleed Anatomical Rating Scale (MARS) ${ }^{11}$ and were counted by a single reviewer, excluding cases with gross structural lesions (eg, large parenchymal hematoma, infarct, or surgical cavity) that may limit the reliability of the microhemorrhage counts. A subset of 20 
randomly selected cases was evaluated by a second reviewer to determine interrater reliability of the microhemorrhage counts.

In the head-to-head analysis, all cases in which either reviewer identified an abnormality on either of the 2 conventional susceptibility sequences in the individual analysis were presented to the reviewers in a separate blinded review, with the wave-SWI and conventional susceptibility-weighted sequence simultaneously displayed on the left and right halves of the screen, labeled image A and image B. The order of the cases and the screen position of the sequences were randomized. The raters compared and scored the 2 sequences for each of the following variables: visualization of pathology (primary outcome), the presence of artifacts (including motion, signal drop-out, and parallel imaging artifacts), and overall diagnostic quality. A predefined 5-point scale was used, in which positive numbers favored the sequence on the right and negative numbers favored the sequence on the left side of the screen (On-line Table 3). Disagreements between readers were adjudicated by a third neuroradiologist (P.W.S.) with $>20$ years of experience.

\section{Statistical Analysis}

In the individual analysis, we used the McNemar test for comparison of dichotomous variables and the Wilcoxon signed rank test for comparison of ordinal variables between sequences. For motion grading, scores of 0 and 1 (ie, "no motion" and "mild motion that is not clinically relevant") were combined in a single category, because this distinction was not clinically meaningful. In the head-to-head analysis, we tested for superiority of waveSWI compared with T2*WI GRE and for noninferiority of waveSWI compared with standard SWI. This approach was selected a priori on the basis of our hypothesis that the wave-SWI was comparable with the standard SWI but superior to the T2*-weighted GRE images. For superiority testing, we compared the ordinal radiologists' scores using the Wilcoxon signed rank test with the null hypothesis $\left(\mathrm{H}_{0}\right)$ of no difference between sequences. For noninferiority testing, ${ }^{12}$ a noninferiority margin $(\Delta)$ of $15 \%$ was chosen, with the null hypothesis $\left(\mathrm{H}_{0}\right)$ that the proportion of cases in which standard SWI was preferred over wave-SWI was $>15 \%$. We used the $z$ statistic to calculate the probability of the standard sequence being preferred over the wave-SWI sequence in $>15 \%$ of cases $\left(\mathrm{H}_{0}>\Delta\right)$, with a type I error rate $(\alpha)$ of 0.05 . We also calculated the upper bound of the $95 \%$ confidence interval for the proportion of cases in which the standard SWI was preferred over wave-SWI (ie, the critical value, $\left.\mathrm{P}_{\text {critical }}\right){ }^{13}$ The required sample size was estimated as described by Cohen $^{14}$ for a single proportion (the proportion of cases in which visualization of pathology was preferred on standard SWI over wave-SWI), for an effect size of 0.15 , a type I error rate $(\alpha)$ of 0.05 , and a power $(1-\beta)$ of 0.90 . According to this calculation, a minimum of 63 cases with abnormal findings was required. For dichotomous variables, interrater agreement was evaluated using the unweighted Cohen $\kappa$ coefficient.

For ordinal variables, interrater agreement was reported using the quadratically weighted Cohen $\kappa$, to disproportionately penalize larger disagreements. For the numeric microhemorrhage counts, interrater agreement was reported using the intraclass correlation coefficient. Agreement was interpreted according to
Landis and Koch. ${ }^{15}$ We applied a Bonferroni correction for 5 comparisons (the presence of hemorrhage, motion artifacts, visualization of pathology, artifacts, and overall diagnostic quality), with a corrected threshold for a statistical significance of .05/ $5=.01$. We also performed exploratory univariate testing evaluation for the possible effect of age and study indication on motion scores. For age, we calculated the Pearson correlation coefficient between patient age and the motion score. For indication, we performed a multinomial logistic regression with the indication as the independent variable and motion score as the dependent variable. All statistical calculations were performed using R statistical and computing software, Version 3.4 .3 (http://www.r-project. $\operatorname{org} /)$.

\section{RESULTS}

Wave-SWI was successfully acquired, reconstructed, and evaluated in all 246 cases, including 139 (56.5\%) with comparison with T2*W GRE (Fig 1) and 107 (43.5\%) with comparison with standard SWI (Fig 2). Abnormalities were identified in 85 cases with comparison with $\mathrm{T} 2^{\star} \mathrm{W}$ GRE and 65 cases with comparison with standard SWI, which were included in the head-to-head analysis. In the individual analysis, interrater agreement ranged from moderate to almost perfect $(\kappa=0.85$ [95\% CI, $0.80-0.89$ ] for presence of hemorrhage, 0.52 for motion [ $95 \%$ CI, 0.13-0.90], and 0.49 [95\% CI, 0.35-0.63] for diagnostic quality). Interrater agreement for microhemorrhage counts according to the MARS was almost perfect (intraclass correlation coefficient $=0.84[95 \%$ CI, 0.64-0.93] for infratentorial, 0.95 [95\% CI, 0.89-0.98] for deep, and 0.98 [95\% CI, 0.96-0.99] for lobar microhemorrhages). In the head-to-head analysis, interrater agreement ranged from moderate to substantial ( $\kappa=0.73$ [95\% CI, 0.44-1.00] for evaluation of pathology, 0.63 [95\% CI, 0.37-0.93] for artifacts, 0.47 [95\% CI, 0.01-1.00] for diagnostic quality). There was a weak positive correlation between patient age and motion score $(r=0.289, P<.01)$. There was no significant effect of clinical indication on motion scores.

\section{T2*W GRE $\times$ Wave-SWI Comparison}

In the individual analysis, hemorrhage was detected more frequently using wave-SWI than $\mathrm{T} 2^{\star} \mathrm{W}$ GRE $(P<.001$, On-line Table 4). In 21 cases (15.1\%), hemorrhage was identified only on the wave-SWI sequence. These cases included 15 cases of microhemorrhage, 3 cases of parenchymal hemorrhage $(>10 \mathrm{~mm}), 2$ cases of postoperative extra-axial hemorrhage, and 1 case of intraventricular hemorrhage. More microhemorrhages were detected using wave-SWI compared with T2*W GRE $(P<.01$ for infratentorial, $P=.01$ for deep, and $P<.001$ for lobar microhemorrhages; On-line Table 4). Motion artifacts were rated less severe on $\mathrm{T} 2{ }^{*} \mathrm{~W}$ GRE compared with wave-SWI $(P<.001$, On-line Table 4); however, there was no significant difference in the number of cases that were rated as nondiagnostic ( 7 cases with $\mathrm{T} 2{ }^{*} \mathrm{~W}$ GRE versus 6 cases with wave-SWI).

The results of the head-to-head comparison of wave-SWI and $\mathrm{T} 2^{\star} \mathrm{W}$ GRE are shown in Fig 3. Wave-SWI was rated superior to $\mathrm{T} 2{ }^{\star} \mathrm{W}$ GRE for visualization of pathology, the presence of artifacts, overall diagnostic quality, and visualization of normal anatomic structures (all $P<.001$ ). 


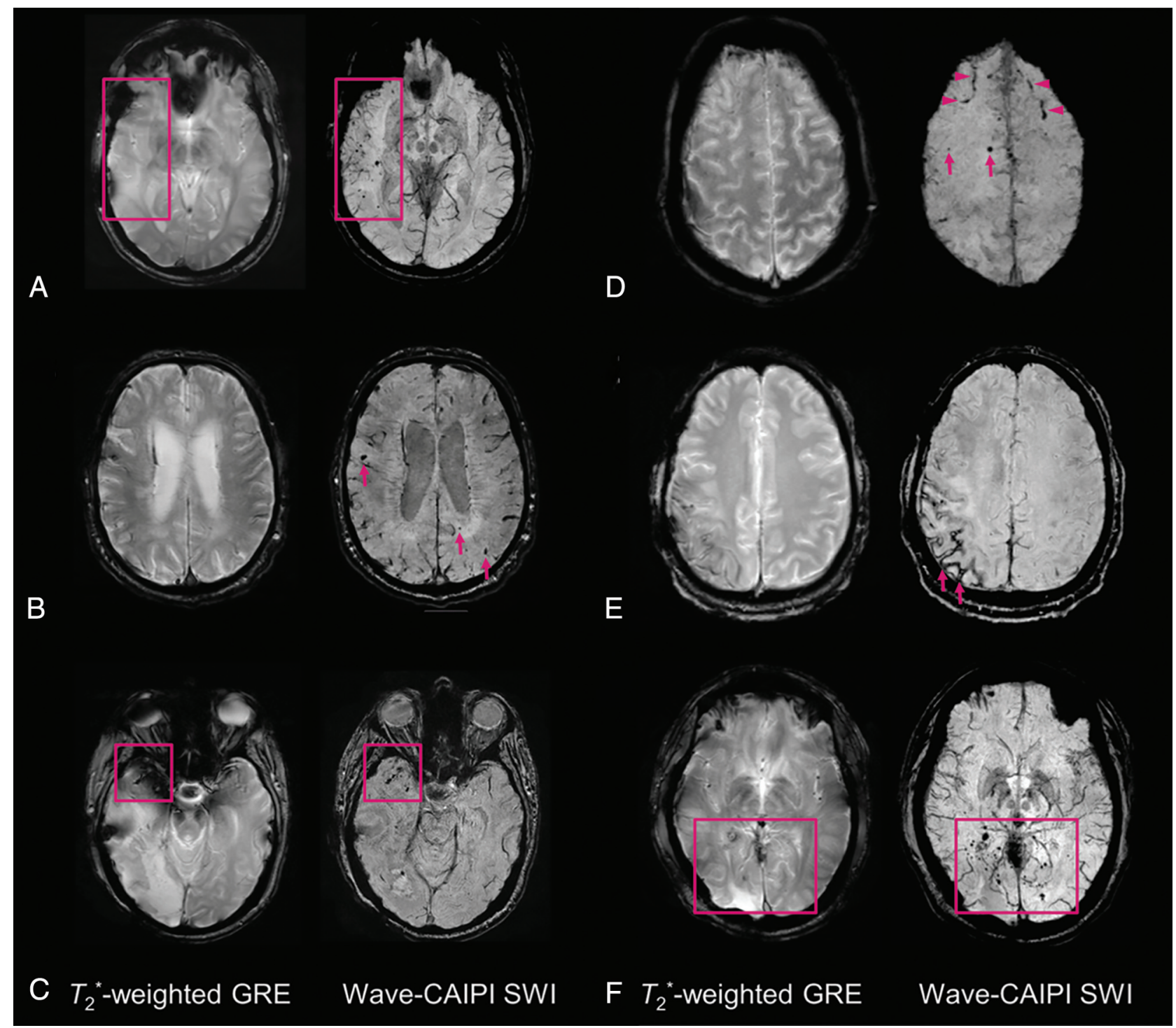

FIG 1. Representative images comparing T2*W GRE and wave-SWI. A, Small hemorrhagic foci in the right temporal lobe are clearly seen on wave-SWI but are not well-visualized on T2*W GRE. These abnormalities fall within the radiation field of a treated right temporal lobe oligoastrocytoma and are presumed to reflect sequelae of radiation-induced vasculopathy. $B$, Multiple cerebral microhemorrhages in a 72year-old man with history of cardiopulmonary bypass for aortic valve replacement and ascending aortic aneurysm repair, some of which are better seen on wave-SWI and some of which are seen only on wave-SWI (arrows). C, Hemorrhagic foci within a right anterior temporal lobe glioblastoma are clearly visualized on wave-SWI but obscured on T2*W GRE due to signal drop-out artifacts on the GRE sequence. D, Scattered foci of parenchymal (arrows) and subarachnoid (arrowheads) hemorrhage in the bilateral frontal lobes are better visualized on wave-SWI than T2*W GRE. E, Superficial hemosiderosis in the right frontoparietal region is better visualized on wave-SWI than T2*W GRE (arrows) in a patient with a history of multiple craniotomies for a recurrent anaplastic astrocytoma. $F$, Scattered foci of posterior predominant susceptibility effect are well-visualized on wave-SWI and not well-seen on T2*W GRE (box), in a 58-year-old man with remote radiation therapy to a posterior fossa atypical meningioma. The findings were presumed to reflect postradiation changes (microhemorrhages and/or small cavernous malformations).

\section{Standard SWI $\times$ Wave-SWI Comparison}

In the individual analysis, there was no significant difference in the presence or number of hemorrhages identified on the waveSWI and standard SWI sequences (On-line Table 4). Motion artifacts were rated less severe with wave-SWI compared with standard SWI $(P<.01)$, with twice as many cases receiving a motion score of 3 (severe motion that may obscure major findings) on the standard SWI sequence (21 cases, 19.6\%) compared with the wave-SWI sequence ( 9 cases, $9.3 \%$ ). More cases were rated nondiagnostic on standard SWI (9 cases, 8.4\%) than wave-SWI (3 cases, $2.8 \%)$; however, the difference was not statistically significance $(P=.08)$.

The results of the head-to-head comparison and the associated noninferiority testing are shown in Fig 4. Wave-SWI was noninferior to standard SWI for visualization of pathology $(P<.001)$, artifacts $(P<.01)$, and overall diagnostic quality $(P<.01)$ with a $15 \%$ noninferiority margin. The critical value for each noninferiority test is shown in Fig 4, corresponding to the upper bound of a 95\% confidence interval on the proportion of cases in which standard SWI is preferred over wave-SWI. This 


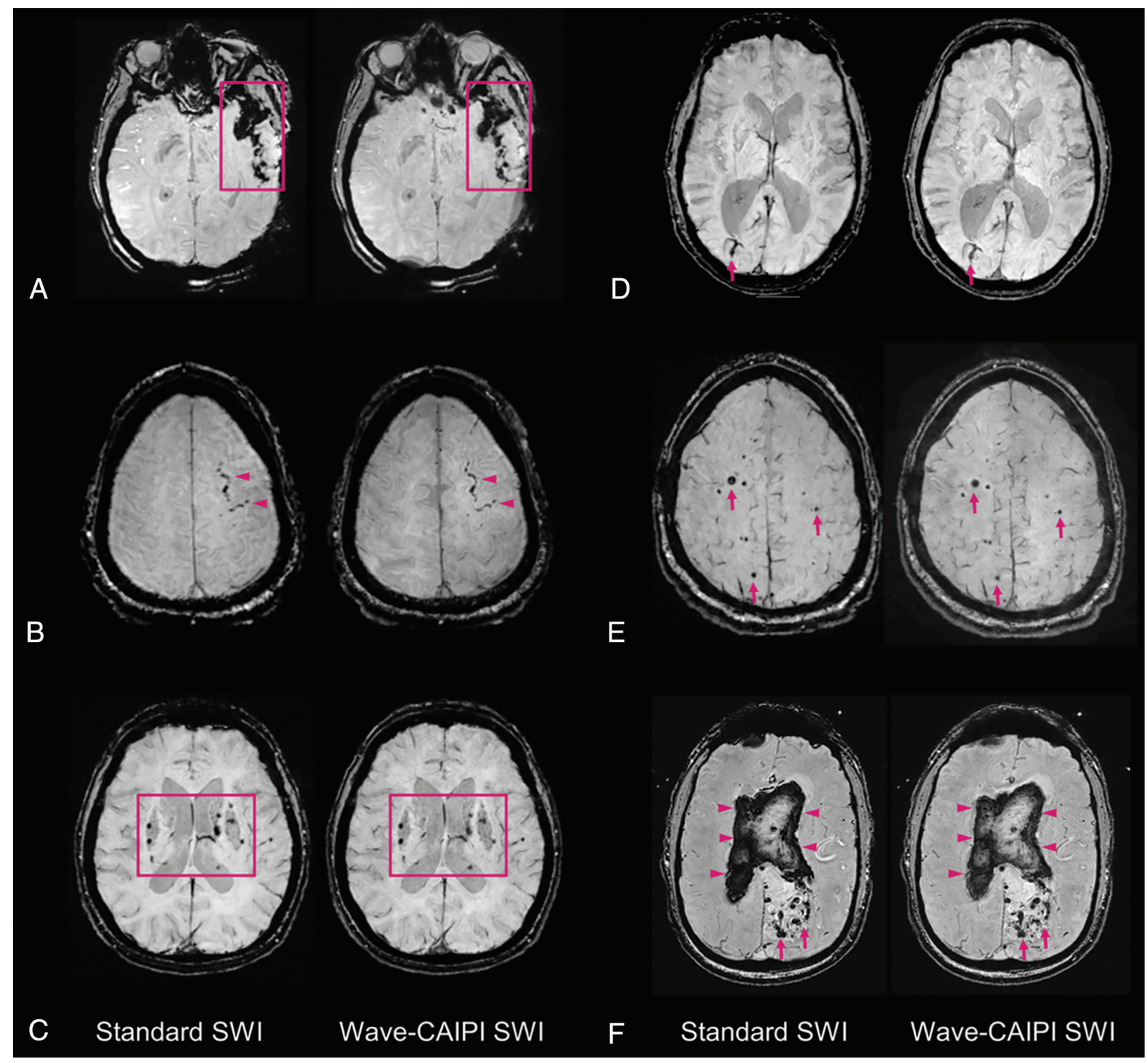

FIG 2. Representative images comparing standard SWI and wave-SWI. A, Extensive susceptibility effect in the left temporal region in a 33-yearold woman with a history of trauma, corresponding to a combination of parenchymal contusion and subdural and subarachnoid hemorrhage. $B$, Focal subarachnoid hemorrhage in the left superior frontal sulcus (arrowheads). C, Scattered microhemorrhages throughout the bilateral basal ganglia in a patient with chronic poorly controlled hypertension. $D$, Incidental finding of a right occipital lobe developmental venous anomaly (arrows). E, Scattered foci of susceptibility effect in a patient with familial multiple cavernous malformations (arrows). F, Serpiginous foci of susceptibility effect in the left occipital region corresponding to an arteriovenous malformation (arrows), with associated rupture and extensive diffuse intraventricular hemorrhage (arrowheads). In all cases, visualization of the pathology was rated equivalent (score of 0 , On-line Table 3) by both interpreting radiologists.

upper bound ranged from $4 \%$ for visualization of pathology to $11 \%$ for artifacts and overall diagnostic quality.

\section{DISCUSSION}

This study compared a highly accelerated wave-SWI sequence with 2 commonly used alternative sequences in terms of technical feasibility, visualization of pathology including hemorrhage, and overall diagnostic quality. We included consecutive MR imaging examinations obtained for a wide range of indications to understand the implications of adopting this sequence for routine clinical brain imaging.
Compared with standard $\mathrm{T} 2{ }^{\star} \mathrm{W}$ GRE, wave-SWI provided superior visualization of pathology and overall diagnostic quality, with acquisition times that were approximately 1.5 times faster than standard $\mathrm{T} 2{ }^{*} \mathrm{~W}$ GRE when the 20 -channel coil was used and 2 times faster when the 32-channel coil was used. The differences in diagnostic quality were clinically relevant, including 21 cases $(15.1 \%)$ in which hemorrhage was seen only on wave-SWI. With increasing use of MR imaging in the emergency setting, ${ }^{16}$ the ability to rapidly detect acute pathology including hemorrhage is particularly desirable. Detection of even a small number of microhemorrhages is often clinically important. For example, 


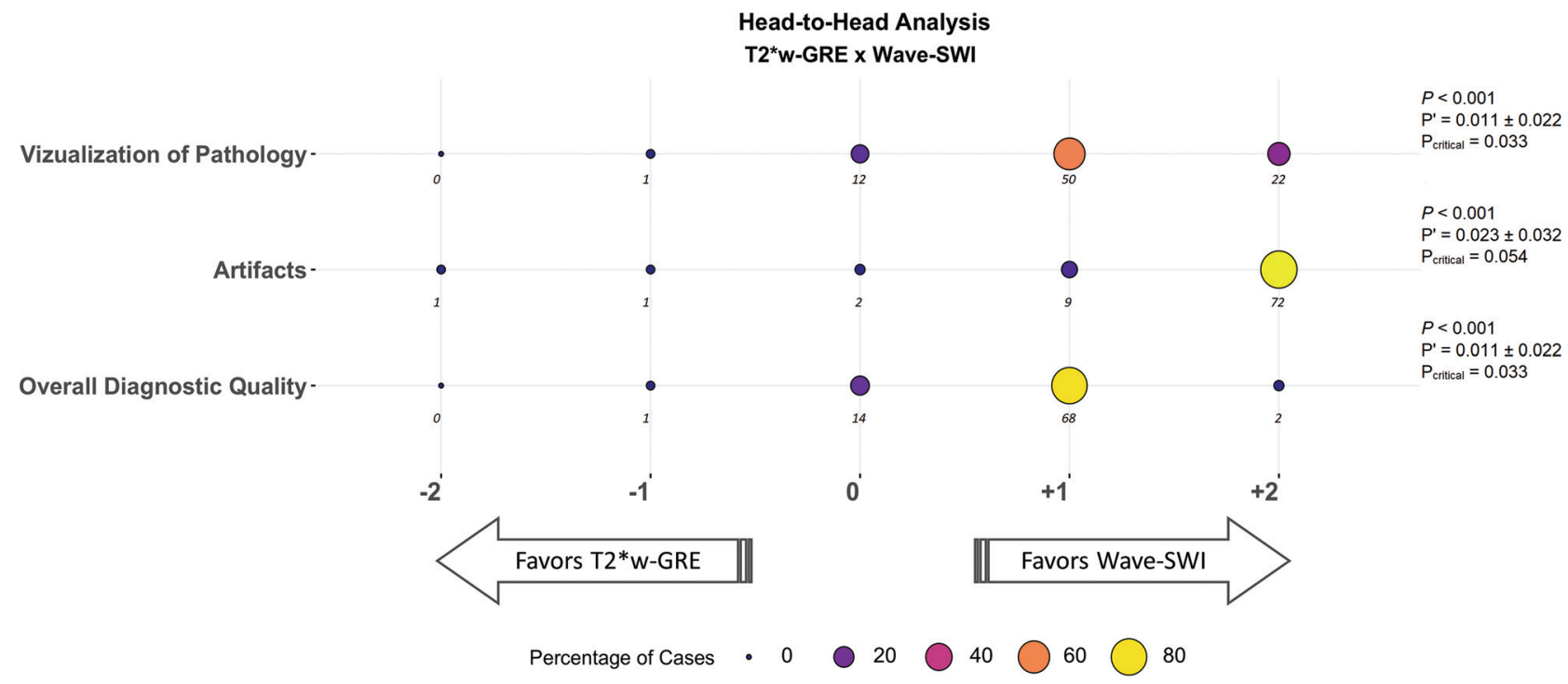

FIG 3. Balloonplot showing the results of the head-to-head comparison of T2*W GRE and wave-SWI. The size and color of each circle represent the percentage of cases that were assigned a given score, from a total of 85 cases with abnormal findings. The actual number of cases receiving a given score is indicated below each circle. Negative scores (left) favor T2*WI GRE, and positive scores (right) favor wave-SWI. The proportion of cases in which T2*W GRE was preferred over wave-SWI ( $\left.P^{\prime}\right)$ and the $95 \%$ confidence interval for this proportion are indicated at the right of the figure. The critical value $\left(P_{\text {critical }}\right)$ is also provided, corresponding to the upper bound of the $95 \%$ confidence interval for $P^{\prime}$. Superiority testing was performed as described in the Materials and Methods, and the corresponding $P$ values are shown in the figure. Wave-SWI was superior to T2*W GRE for the 3 variables evaluated.

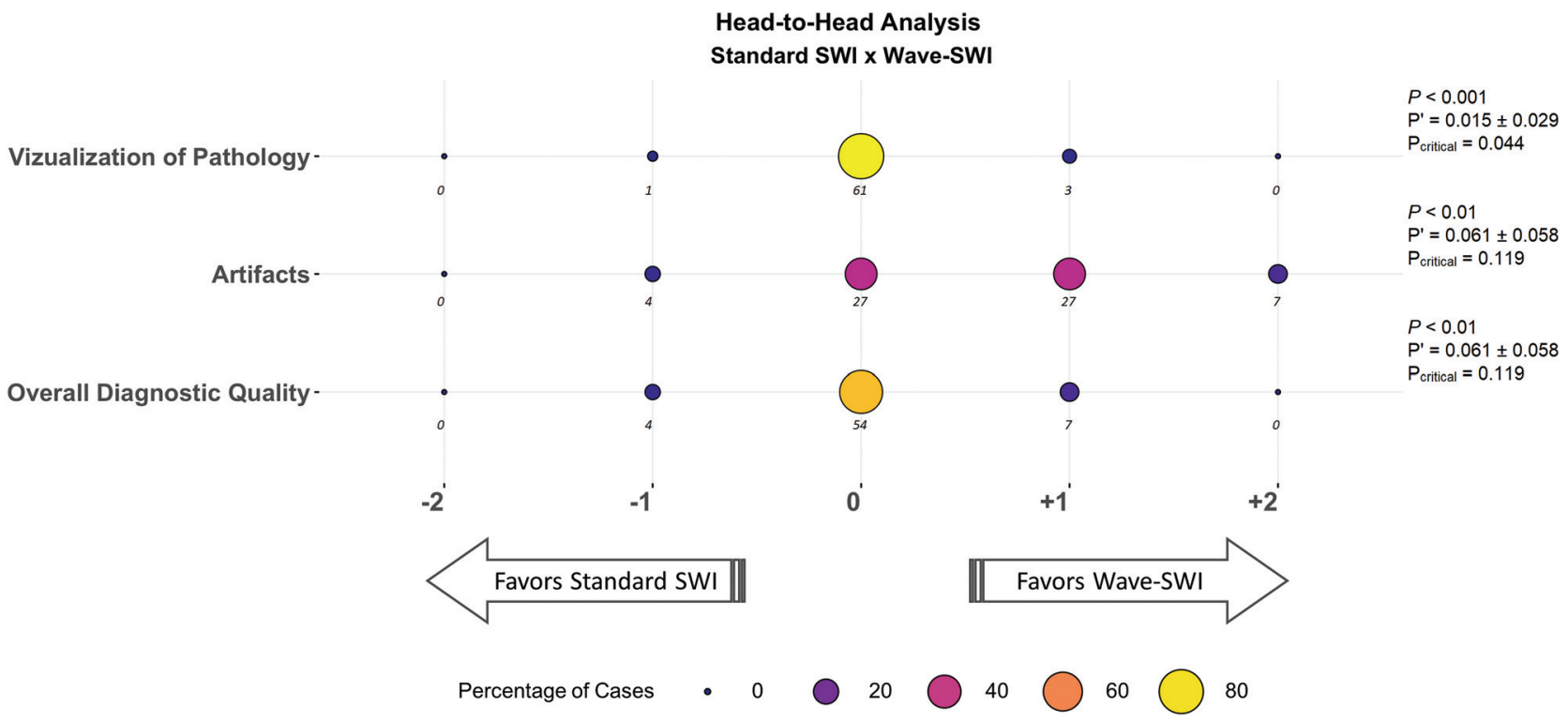

FIG 4. Balloonplot showing the results of the head-to-head comparison of standard SWI and wave-SWI. The size and the color of each circle represent the percentage of cases that were assigned a given score, from a total of 65 cases with abnormal findings. The actual number of cases receiving a given score is indicated below each circle. Negative scores (left) favor standard SWI, and positive scores (right) favor wave-SWI. The proportion of cases in which T2*WI GRE was preferred over wave-SWI ( $\left.P^{\prime}\right)$ and the $95 \%$ confidence interval for this proportion are indicated at the right of the figure. The critical value $\left(P_{\text {critical }}\right)$ is also provided, corresponding to the upper bound of the $95 \%$ confidence interval for $P^{\prime}$. Noninferiority testing was performed as described in the Materials and Methods, and the corresponding $P$ values are shown in the figure. WaveSWI was noninferior to standard SWI for the 3 variables evaluated.

in patients who have undergone anticoagulation with a history of atrial fibrillation and recent stroke, the presence of microhemorrhages is associated with intracranial hemorrhage risk and may inform anticoagulation decisions. ${ }^{17}$ Artifacts were rated more severe on $\mathrm{T} 2{ }^{\star} \mathrm{W}$ GRE, likely due to thicker slices resulting in greater through-plane dephasing and signal drop-out near the skull base (Fig 1C). Motion was rated as less severe on $\mathrm{T}^{*} \mathrm{~W}$ GRE, likely due to the intrinsically lower motion sensitivity of multisection $2 \mathrm{D}$ versus $3 \mathrm{D}$ sequences. ${ }^{18}$ Thus, the reduced motion sensitivity of T2*W GRE (and the even faster echo-planar 
imaging-based GRE sequences) may provide advantages over wave-SWI in the setting of extreme motion. Our findings suggest that wave-SWI could replace $\mathrm{T} 2{ }^{*} \mathrm{~W}$ GRE for most indications, providing superior visualization of pathology with a 1.5- to 2-fold decrease in scan time, encouraging greater adoption of SWI across clinical brain imaging protocols. Wave-SWI also provides additional information over T2*W GRE in the form of filteredphase maps, which can be useful in distinguishing blood products from mineralization. ${ }^{19}$

Wave-SWI was noninferior to standard SWI for visualization of pathology, artifacts, and overall diagnostic quality, with reduced scan time (approximately 3 times faster than standard SWI on the 20-channel coil and 5 times faster on the 32-channel coil) and reduced motion artifacts. Twice as many scans received a motion score of 3 (severe motion that may obscure major findings) using standard SWI, likely a direct consequence of the longer acquisition time. Motion is a common source of image degradation in MR imaging, especially in emergency and inpatient settings, and is associated with substantial institutional cost. ${ }^{20}$ Our findings suggest that wave-SWI could replace standard SWI for most indications, improving the use of $\mathrm{MR}$ imaging resources while reducing motion artifacts and cost associated with repeat sequence attempts or repeat examinations. The decreased scan time of wave-CAIPI may provide synergistic benefits with other prospective ${ }^{21}$ and retrospective $^{22}$ motion-correction strategies. For example, the recently proposed Targeted Motion Estimation and Reduction algorithm uses a joint optimization similar to the autocalibrated wave-CAIPI reconstruction, ${ }^{22}$ and future work incorporating Targeted Motion Estimation and Reduction directly into the joint reconstruction may provide even further reduction in the motion sensitivity of wave-SWI. Comparison of wave-CAIPI with other acceleration strategies based on compressed sensing ${ }^{23}$ and exploiting possible synergies between these approaches would be a valuable area for future study. Further evaluation of multiecho approaches, including the optimal number and spacing of echoes for R2* mapping and quantitative susceptibility mapping, would also be a valuable extension of this work.

Our study has several limitations. First, it is not possible to compare the diagnostic accuracy of wave-SWI and standard SWI in vivo due to the absence of a reference standard. Because of the similar contrast and image quality of these sequences and the variable nature of patient motion and other image artifacts, detection of a small hemorrhage on wave-SWI but not standard SWI does not necessarily imply a false-positive and could alternatively reflect motion artifacts obscuring the hemorrhage on the standard sequence. To validate wave-SWI as an index test in the absence of a reference standard, we followed the approach described by Reitsma et $\mathrm{al}^{24}$ by relating the result of the index test to other clinically relevant characteristics-in our case, the impact on the final clinical diagnosis determined by the interpreting radiologist (On-line Table 3). Under this framework, we demonstrated noninferiority of the wave-SWI sequence with respect to the major factors relevant to the interpreting radiologist in rendering a clinical diagnosis.

Although we used a standardized image-based definition of microhemorrhages, ${ }^{11}$ we did not have CT correlation to confirm the presence of calcification or follow-up imaging to confirm persistence or expansion of hemorrhage, and it is possible that some of the microhemorrhages we identified were, in fact, calcifications or other mimics. Second, the selection of a suitable noninferiority margin for imaging studies is often challenging. Our selection was informed by a review of similar imaging-based noninferiority studies $^{25,26}$ and consensus among our group of neuroradiologists that the new sequence could be considered noninferior if the standard sequence was preferred in fewer than $15 \%$ of cases. Because this threshold is inherently subjective, we also reported the critical value $\left(\mathrm{P}_{\text {critical }}\right)$, equivalent to the upper bound on a $95 \%$ confidence interval for the proportion of cases in which the standard sequence was preferred. The critical value allows us to understand how selection of a different noninferiority margin would impact the results. For example, with a $25 \%$ noninferiority margin, wave-SWI would be deemed noninferior to standard SWI for all of the variables considered $\left(\mathrm{P}_{\text {critical }},<25 \%\right.$ in all cases; Fig 4$)$. With a $5 \%$ noninferiority margin, wave-SWI would be deemed noninferior for visualization of pathology (the primary outcome), but not the remaining variables. Third, although readers were blinded to the acquisition protocol, some features of the images may allow the readers to identify the pulse sequence being evaluated. In the case of wave-SWI versus $\mathrm{T} 2{ }^{\star} \mathrm{W}$ GRE, the difference is visibly apparent. In the case of wave-SWI versus standard SWI, we minimized this possibility by matching the most important parameters that determine image quality and image contrast (including nominal voxel dimensions, section thickness, and effective echo time) as closely as possible within the range of allowable parameter values.

\section{CONCLUSIONS}

Wave-SWI provided superior visualization of pathology and overall diagnostic quality compared with $\mathrm{T} 2{ }^{*} \mathrm{~W}$ GRE and was noninferior to standard SWI with reduced scan time and reduced motion artifacts. Broader clinical application of the wave-CAIPI approach may result in more efficient use of MR imaging resources without the loss of clinically important information.

Disclosures: Maria Gabriela F. Longo-RELATED: Other: Siemens Healthineers, Comments: scholarship for postdoctoral program.* Kawin SetsompopRELATED: Grant: National Institutes of Health, Comments: R01EB020613.* Susie Y. Huang-RELATED: Grant: Siemens Healthineers, Comments: received a research grant from Siemens to support a research fellow to carry out clinical validation of wave-controlled aliasing in parallel imaging technology.* Stephen F. Cauley-RELATED: Grant: National Institutes of Health, Comments: IR01EB020613-01A1, IU01HD087211-01, 5P41EB015896-18, 2R01EB00684709A1*; UNRELATED: Grants/Grants Pending: Siemens, Comments: Artificial Intelligence fast imaging grant.* *Money paid to the institution.

\section{REFERENCES}

1. Nandigam RN, Viswanathan A, Delgado P, et al. MR imaging detection of cerebral microbleeds: effect of susceptibility-weighted imaging, section thickness, and field strength. AJNR Am J Neuroradiol 2009;30:338-43 CrossRef Medline

2. Haacke EM, Mittal S, Wu Z, et al. Susceptibility-weighted imaging: technical aspects and clinical applications, Part 1. AJNR Am J Neuroradiol 2009;30:19-30 CrossRef Medline

3. Mittal S, Wu Z, Neelavalli J, et al. Susceptibility-weighted imaging: technical aspects and clinical applications, Part 2. AJNR Am J Neuroradiol 2009;30:232-52 CrossRef Medline

4. Havsteen I, Ohlhues A, Madsen KH, et al. Are movement artifacts in magnetic resonance imaging a real problem? A narrative review. Front Neurol 2017;8:1-8 CrossRef Medline 
5. Munn Z, Pearson A, Jordan Z, et al. Patient anxiety and satisfaction in a magnetic resonance imaging department: initial results from an action research study. J Med Imaging Radiat Sci 2015;46:23-29 CrossRef Medline

6. Bilgic B, Gagoski BA, Cauley SF, et al. Wave-CAIPI for highly accelerated 3D imaging. Magn Reson Med 2015;73:2152-62 CrossRef Medline

7. Cauley SF, Setsompop K, Bilgic B, et al. Autocalibrated wave-CAIPI reconstruction; joint optimization of k-space trajectory and parallel imaging reconstruction. Magn Reson Med 2017;78:1093-99 CrossRef Medline

8. Bilgic B, Xie L, Dibb R, et al. Rapid multi-orientation quantitative susceptibility mapping. Neuroimage 2016;125:1131-41 CrossRef Medline

9. Bilgic B, Ye H, Wald LL, et al. Simultaneous time interleaved multislice (STIMS) for rapid susceptibility weighted acquisition. Neuroimage 2017;155:577-86 CrossRef Medline

10. Conklin J, Cauley S, Setsompop K, et al. Optimization and Clinical Evaluation of Wave-CAIPI Susceptibility-Weighted Imaging (SWI) for Detection of Intracranial Hemorrhage. In: Proceedings of the Annual Meeting of the Radiological Society of North America, Chicago, Illinois. November 25-30, 2018; SSE24-04

11. Gregoire SM, Chaudhary UJ, Brown MM, et al. The Microbleed Anatomical Rating Scale (MARS): reliability of a tool to map brain microbleeds. Neurology 2009;73:1759-66 CrossRef Medline

12. Ahn S, Park SH, Lee KH. How to demonstrate similarity by using noninferiority and equivalence statistical testing in radiology research. Radiology 2013;267:328-38 CrossRef Medline

13. Lakens D, Scheel AM, Isager PM. Equivalence testing for psychological research: a tutorial. Adv Advances in Methods and Practices in Psychological Science 2018;1:259-69 CrossRef

14. Cohen J. Statistical Power Analysis for the Behavioral Sciences. 2nd ed. Hillsdale: L. Erlbaum Associates; 1988

15. Landis JR, Koch GG. The measurement of observer agreement for categorical data. Biometrics 1977;33:159-74 CrossRef Medline

16. Redd V, Levin S, Toerper M, et al. Effects of fully accessible magnetic resonance imaging in the emergency department. Acad Emerg Med 2015;22:741-49 CrossRef Medline

17. Wilson D, Ambler G, Shakeshaft C, et al. Cerebral microbleeds and intracranial haemorrhage risk in patients anticoagulated for atrial fibrillation after acute ischaemic stroke or transient ischaemic attack (CROMIS-2): a multicentre observational cohort study. Lancet Neurol 2018;17:539-47 CrossRef Medline

18. Runge VM, Wood ML, Kaufman DM, et al. FLASH: clinical threedimensional magnetic resonance imaging. Radiographics 1988;8:94765 CrossRef Medline

19. Yamada N, Imakita S, Sakuma T, et al. Intracranial calcification on gradient-echo phase image: depiction of diamagnetic susceptibility. Radiology 1996;198:171-78 CrossRef Medline

20. Andre JB, Bresnahan BW, Mossa-Basha M, et al. Toward quantifying the prevalence, severity, and cost associated with patient motion during clinical MR examinations. J Am Coll Radiology 2015;12:689-95 CrossRef Medline

21. Callaghan MF, Josephs O, Herbst M, et al. An evaluation of prospective motion correction (PMC) for high resolution quantitative MRI. Front Neurosci 2015;9:97 CrossRef Medline

22. Haskell MW, Cauley SF, Wald LL. TArgeted Motion Estimation and Reduction (TAMER): Data consistency based motion mitigation for MRI using a reduced model joint optimization. IEEE Trans Med Imaging 2018;37:1253-65 CrossRef Medline

23. Wu B, Li W, Guidon A, et al. Whole brain susceptibility mapping using compressed sensing. Magn Reson Med 2012;67:137-47 CrossRef Medline

24. Reitsma JB, Rutjes AWS, Khan KS, et al. A review of solutions for diagnostic accuracy studies with an imperfect or missing reference standard. J Clin Epidemiol 2009;62:797-806 CrossRef Medline

25. Lee SJ, Park SH, Kim AY, et al. A prospective comparison of standard-dose CT enterography and $50 \%$ reduced-dose CT enterography with and without noise reduction for evaluating Crohn disease. AJR Am J Roentgenol 2011;197:50-57 CrossRef Medline

26. Schaefer PJ, Boudghene FP, Brambs HJ, et al. Abdominal and iliac arterial stenoses: comparative double-blinded randomized study of diagnostic accuracy of 3D MR angiography with gadodiamide or gadopentetate dimeglumine. Radiology 2006; 238:827-40 CrossRef Medline 